\title{
The effects of the cross-entropy stopping criterion and quadrature amplitude modulation on iterative turbo decoding performance
}

Roslina Mohamad', Mohamad Yusuf Mat Nasir², Nuzli Mohamad Anas ${ }^{3}$

${ }^{1,2}$ Wireless High Speed Network Research Group (WHiSNet), School of Electrical Engineering, College of Engineering, Universiti Teknologi MARA, Selangor, Malaysia

${ }^{1,2}$ College of Engineering, Universiti Teknologi MARA, Selangor, Malaysia

${ }^{3}$ Wireless Innovation, MIMOS Berhad, Wilayah Persekutuan, Malaysia

\begin{abstract}
Article Info
Article history:

Received Jun 1, 2021

Revised Aug 4, 2021

Accepted Aug 10, 2021

\section{Keywords:}

Cross-entropy

Iterative decoding

Quadrature amplitude

modulation

Stopping criteria

Turbo codes

ABSTRACT

One of the most often-used stopping criteria is the cross-entropy stopping criterion (CESC). The CESC can stop turbo decoder iterations early by calculating mutual information improvements while maintaining bit error rate (BER) performance. Most research on iterative turbo decoding stopping criteria has utilised low-modulation methods, such as binary phase-shift keying. However, a high-speed network requires high modulation to transfer data at high speeds. Hence, a high modulation technique needs to be integrated into the CESC to match its speed. Therefore, the present paper investigated and analysed the effects of the CESC and quadrature amplitude modulation (QAM) on iterative turbo decoding. Three thresholds were simulated and tested under four situations: different code rates, different QAM formats, different code generators, and different frame sizes. The results revealed that in most situations, the use of CESC is suitable only when the signal-to-noise ratio (SNR) is high. This is because the CESC significantly reduces the average iteration number (AIN) while maintaining the BER. The CESC can terminate early at a high SNR and save more than $40 \%$ AIN compared with the fixed stopping criterion. Meanwhile, at a low SNR, the CESC fails to terminate early, which results in maximum AIN.
\end{abstract}

This is an open access article under the CC BY-SA license.

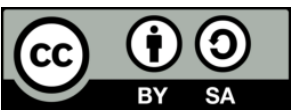

Corresponding Author:

Roslina Mohamad

Wireless High Speed Network Research Group (WHiSNet)

School of Electrical Engineering, College of Engineering

Universiti Teknologi MARA

40450 Shah Alam, Selangor, Malaysia

Email: roslina780@uitm.edu.my

\section{INTRODUCTION}

Error-correcting coding, also known as channel coding, is critical to achieving optimum efficiency in information transmission by lowering the likelihood of errors in digital communications systems [1], [2]. A channel encoder systematically adds redundant data and transforms bit sequences of information into encoded bit sequences. A channel decoder can correct a received signal's redundant bits that have been distorted by the receiver's noisy channel [3], [4]. This channel coding technique is called forward error correction (FEC).

Turbo codes are a leading candidate for FEC codes when superior output is required near the Shannon capacity limit [5], [6]. Turbo codes have been used widely in various areas, including digital video broadcasting, deep-space and satellite communications, to improve the chance of obtaining error-free data 
[7], [8]. The maximum a posteriori (MAP) probability algorithm and iterative turbo decoding are two important components in the success of turbo codes [9], [10]. However, using a MAP algorithm to accomplish iterative turbo decoding necessitates additional computations, significant memory costs, and decoding system delay [11], [12].

Researchers solve this problem by developing stopping criteria and increasing turbo codes' performance [13]-[16]. Specifically, stopping criteria help reduce the iteration number in iterative turbo decoding while preserving turbo code efficiency [17], [18]. Most stopping criteria have been developed based on the frame level [7], [9], [14], and bit/window level [11], [12], [16]. Soft-decision-based stopping criteria have become a popular choice for output decisions at the frame level [10], [19], [20].

The average number of bits required to identify an event selected from the set is determined by the cross-entropy between two probability distributions $\mathbf{A}$ and $\mathbf{B}$, over the same underlying set of events, according to information theory. This is especially true when the set's coding method is optimised for an estimated probability distribution A rather than the genuine distribution B. Cross-entropy is used in many applications, such as for determining stopping criteria in iterative decoding scenarios [13]-[15], defining loss functions in machine learning [21], [22], and optimising classification models [23].

Fowdur et al. [14], [24] proposed the cross-entropy stopping criterion (CESC) to calculate improvements in mutual information to cease turbo decoder iterations. Most of the research on iterative turbo decoding stopping criteria have utilised the binary phase-shift keying (BPSK) modulation technique [6], [7], [11], [15], which is the simplest form of phase-shift keying. High-speed networks such as long-term evolution (LTE) networks and those based on fifth-generation $(5 \mathrm{G})$ technology require high modulation (For example, eight phase-shift keyings (8PSK), 16 phase-shift keyings (16PSK), 16-QAM, 32-QAM, and 64-QAM) to transfer data at a sufficient speed [14], [16], [25]. A higher modulation needs to be integrated into stopping criteria to meet the speed requirements of current LTE applications [14], [16].

This paper is intended to address the lack of information about the performance of CESC and high modulation via iterative turbo decoding [26]. Hence, this paper describes the effects of CESC and QAM on iterative turbo decoding performance. Three predefined thresholds of CESC were tested under four situations: different code rates, different QAM formats, different code generators, and different frame sizes. The CESC's performance was assessed using the bit error rate (BER) and average iteration number (AIN).

The content of this paper is organised in the following manner. The CESC and its algorithm are discussed in Section 2. In Section 3, the simulation parameters and the methodology used in the research are described. In Section 4, the effects and performances of the CESC with QAM on iterative turbo decoding are analysed and compared. Finally, conclusions are drawn in Section 5.

\section{CROSS-ENTROPY STOPPING CRITERION}

Figure 1 illustrates the process for the early termination of an iterative turbo decoder using the CESC. The CESC is between decoder two and the deinterleaver. The outputs from the first turbo encoder are decoded by decoder 1 , which are the systematic input bit of the decoder, and the initial parity input bit of the decoder $a_{b}^{s}, a_{b, 1}^{p}$.

The following is an explanation of the iterative turbo decoder with a CESC: At the $i$ th iteration and the $b$ th discrete-time index, a posteriori log-likelihood ratio (LLR), $L_{l, n}^{i}\left(x_{b}\right)$, and extrinsic information LLR $L_{e, n}^{i}\left(x_{b}\right)$ are delivered by the $n$th component decoder. Here, $n=1,2$ is given by (1) and (2).

$$
\begin{aligned}
& L_{l, 1}^{i}\left(x_{b}\right)=L_{e, 2}^{i-1}\left(x_{b}\right)+a_{b}+L_{e, 1}^{i}\left(x_{b}\right) \\
& L_{l, 2}^{i}\left(x_{b}\right)=L_{e, 1}^{i}\left(x_{b}\right)+a_{b}+L_{e, 2}{ }^{i}\left(x_{b}\right)
\end{aligned}
$$

The functions $L_{e, 2}^{i}\left(x_{b}\right)$ and $L_{l, 2}^{i}\left(x_{b}\right)$ are input into the CESC. In CESC, the $i$ th iteration is compared to the maximum number of iterations $\left(i_{\max }\right)$. If the condition is not fulfiled, the online CE threshold $(T(i))$ is calculated,

$$
T(i) \approx \sum_{b} \frac{\left|\Delta L_{e, 2}^{i}\left(x_{b}\right)\right|^{2}}{e^{\left|L_{l, 1}^{i}\left(x_{b}\right)\right|}}
$$


where $\Delta L_{e, 2}^{i}\left(x_{b}\right)$,

$$
\Delta L_{e, 2}^{i}\left(x_{b}\right)=L_{l, 2}^{i}\left(x_{b}\right)-L_{l, 1}^{i}\left(x_{b}\right)=L_{e, 2}^{i}\left(x_{b}\right)-L_{e, 2}^{i-1}\left(x_{b}\right)
$$

the iteration is stopped if $T(i)$ is in the thresholds predefined in (5),

$$
T(i)<\left(10^{-2} \sim 10^{-4} T(1)\right.
$$

if the condition is not fulfiled, then $L_{e, 2}^{i}\left(x_{b}\right)$ is returned to decoder 1 , and the process is repeated until $i_{\max }$ is achieved. Otherwise, when $i_{\max }$ is met, the deinterleaver/final output $\left(x_{b}\right)$ can be estimated from $L_{l, 2}^{i}\left(x_{b}\right)$, as shown by (6),

$$
x_{b}=\operatorname{sign}\left(L_{l, 2}^{i}\left(x_{b}\right)\right)=\left\{\begin{array}{ccc}
0 & \text { if } & L_{l, 2}^{i}\left(x_{b}\right)<=0 \\
1 & \text { if } & L_{l, 2}^{i}\left(x_{b}\right)>0
\end{array}\right.
$$

the final turbo decoder output can be written in the following sequence,

$$
\boldsymbol{x}=\left\{x_{b}\right\}_{b=1}^{N}=x_{1}, x_{2}, \ldots, x_{N}
$$

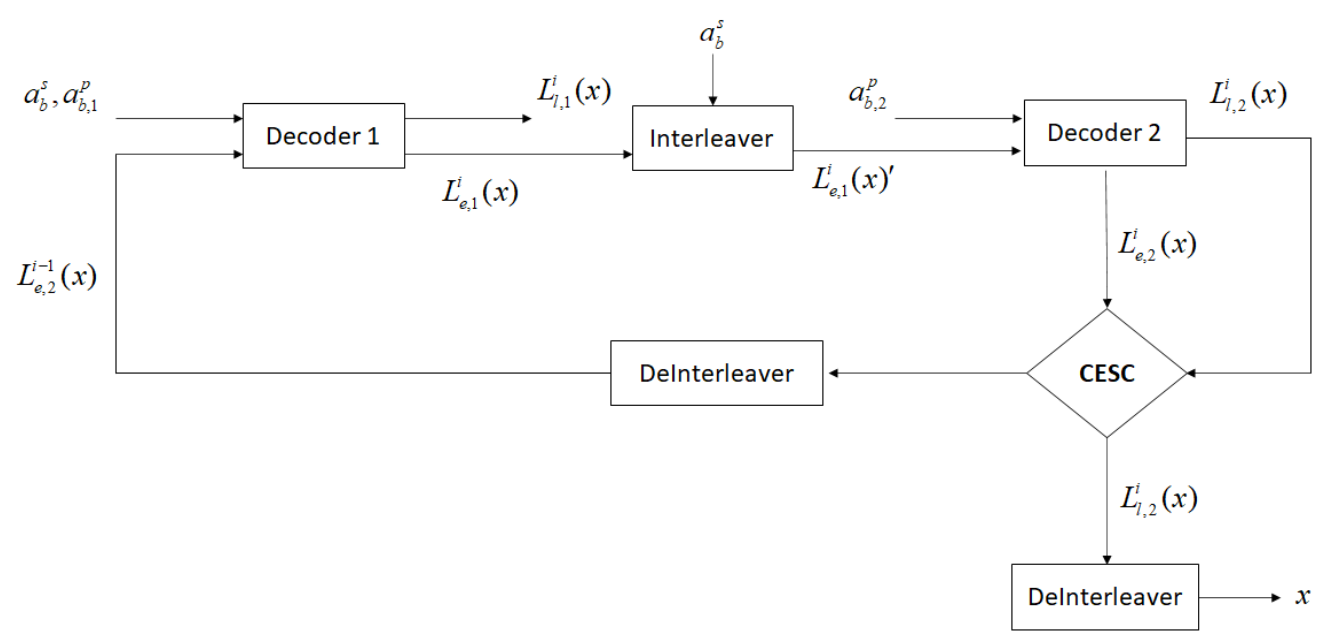

Figure 1. Iterative turbo decoder with a CESC

\section{RESEARCH METHOD}

Table 1 shows the parameters used in the present simulation. The thresholds for CESC were set at 0.1, 0.01, and 0.001. One million random binary data with three different modulations (4-QAM, 8-QAM, and 16-QAM) were applied in the simulation. Meanwhile, the frame size (N) was set to either 1000, 5000, or 10,000 for each data transmission. The turbo code generator (in octal) and code rate values were $(7,5)$, $(17,15),(37,21)$, and $(1 / 2,1 / 3)$. The codes were simulated under the AWGN channel, and the maximum iteration number $\left(I_{\max }\right)$ was set to 8 .

The flowchart in Figure 2 outlines the simulation. The simulation was started with setting up the turbo decoder parameters, such as code generator $(\mathrm{g})$, code rate $(\mathrm{R})$, and frame size $(\mathrm{N})$ as shown in Table 1 . Next, threshold and QAM formats were selected based on the data in Table 1. Then, the turbo decoder with QAM was integrated and simulated and repeated for other turbo code parameters and thresholds for all CESC and QAM formats. Finally, the performances of the turbo decoders with QAM were analysed. 
Table 1. Simulation parameters

\begin{tabular}{cc}
\hline Parameters & Name/ Value \\
\hline CESC threshold & $0.1,0.01,0.001$ \\
Modulation & 4-QAM, 16-QAM, 32-QAM \\
Frame size & $1000(1 \mathrm{~K}), 5000(5 \mathrm{~K}), 10000(10 \mathrm{~K})$ \\
Turbo code generator & $(7,5),(17,15),(37,21)$ \\
Turbo code rate & $1 / 2,1 / 3$ \\
Channel model & AWGN \\
Maximum iteration number & 8 \\
\hline
\end{tabular}

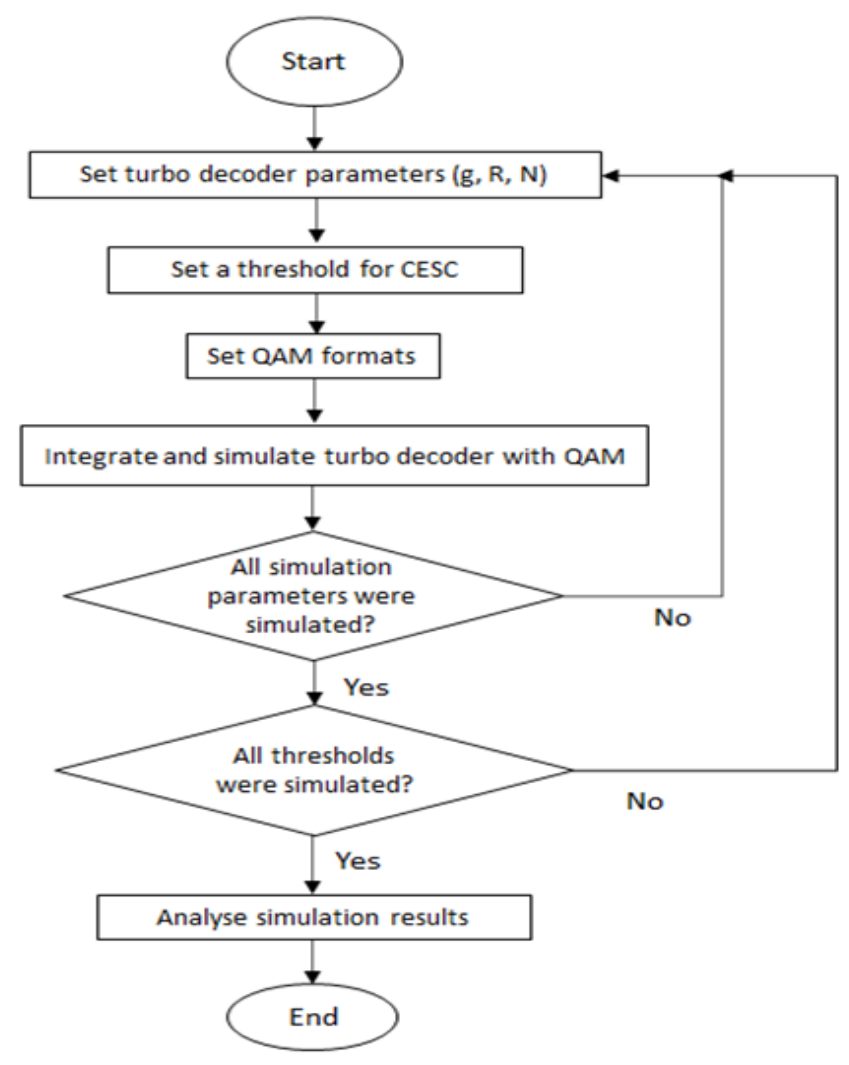

Figure 2. Flowchart for the simulation

\section{RESULTS AND DISCUSSION}

The effects and performances of CESC and QAM with three predefined thresholds were compared and analysed under four situations: different code rates, different QAM formats, different code generators, and different frame sizes as shown in Table 1. Each threshold's performance was analysed based on the BER and AIN.

\subsection{Performance of the CESC with different turbo code rates}

The CESC's AIN and BER performance for coding rates is shown in Figure 3. For the 1/3 code rate, the AIN for threshold values of $0.01,0.001$, and 0.0001 started to decrease at $2 \mathrm{~dB}$. At $4 \mathrm{~dB} \mathrm{~Eb} / \mathrm{No}$, the threshold values of $0.01,0.001$, and 0.0001 can terminate early at 2.8, 2.9, and 3 AIN, respectively. These values represent decreases of around $65 \%, 63.75 \%$, and $62.5 \%$ compared to the fixed stopping criterion (FISC). Moreover, the BER output of the CESC and FISC are nearly the same for threshold values of 0.01 , 0.001 , and 0.0001 .

For the $1 / 2$ code rate, the AIN for threshold values of $0.01,0.001$, and 0.0001 started to decrease at $3 \mathrm{~dB}$. At $4 \mathrm{~dB}$, the threshold values of $0.01,0.001$, and 0.0001 can terminate early at $3.7,3.9$, and 4.1 , respectively. These values represent decreases of $53.75 \%, 51.25 \%$, and $48.75 \%$ compared to FISC. Regarding BER performance, it can be observed that for 0.01 , there is a slight difference in BER starting at $3.5 \mathrm{~dB}$, which increases until $4 \mathrm{~dB}$ at BER $=10^{-4.5}$. For thresholds of 0.001 and 0.0001 , there are slight differences in BER, starting at $3.5 \mathrm{~dB}$ and increasing until $4 \mathrm{~dB}$ at $\mathrm{BER}=10^{-4.7}$. 


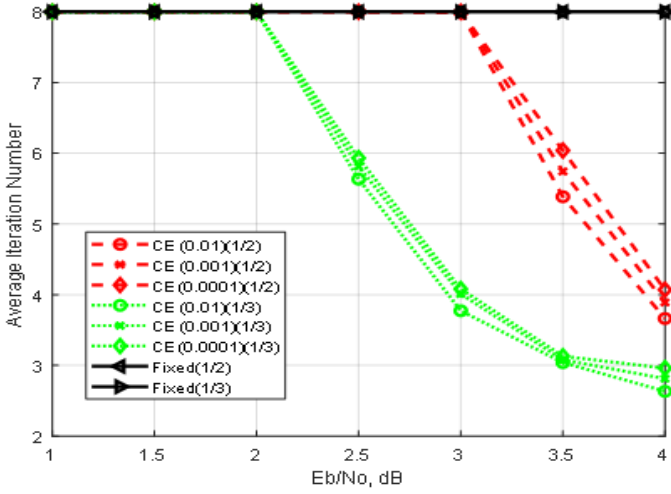

(a)

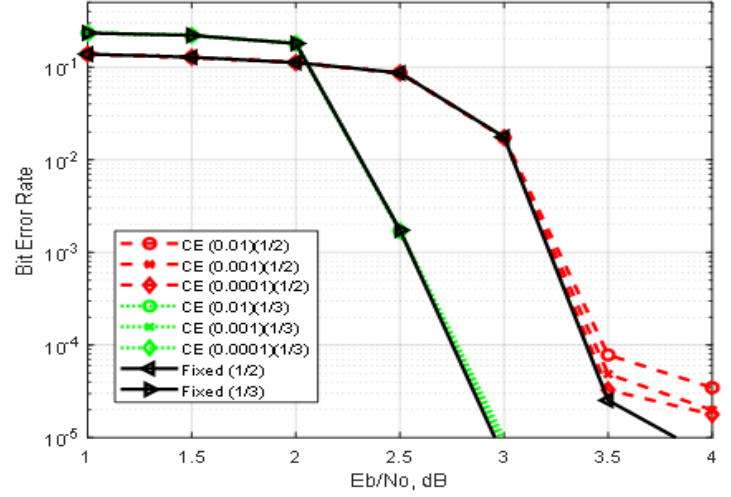

(b)

Figure 3. (a) AIN and (b) BER performances of the CESC with 16-QAM, $g=(37,21)$, and $\mathrm{N}=10 \mathrm{~K}$ for different turbo code rates

\subsection{Performance of the CESC with different QAM formats}

The AIN and BER for CESC for QAM formats of 4-QAM, 16-QAM, and 32-QAM are shown in Figure 4. The 4-QAM graph shows that the AIN start to reduce at $0 \mathrm{~dB}$ for all three threshold values. At $4 \mathrm{~dB}$ $\mathrm{Eb} / \mathrm{No}$, the threshold values of $0.01,0.001$, and 0.0001 can terminate early at two iterations, around $75 \%$ less than FISC. Moreover, slight differences in BER occurred for all three threshold values, starting at $0 \mathrm{~dB}$ and increasing until $0.5 \mathrm{~dB}$ at $\mathrm{BER}=10^{-5}$.

Meanwhile, for 16-QAM, the AIN for threshold values of $0.01,0.001$, and 0.0001 started to reduce at $2 \mathrm{~dB}$. At $4 \mathrm{~dB}$, the threshold value of $0.01,0.001$, and 0.0001 can terminate early at 2.8, 2.9, and 3 AIN, respectively, signifying reductions of around $65 \%, 63.75 \%$, and $62.5 \%$ compared to FISC. The BER performance of the CESC was the same as FISC for all three threshold values.

The 32-QAM indicates that the AIN for threshold values of $0.01,0.001$, and 0.0001 started to reduce at $3 \mathrm{~dB}$. At $4 \mathrm{~dB}$, the threshold values of $0.01,0.001$, and 0.0001 can terminate early at $4.5,4.6$, and 4.8 , respectively - these values are $43.75 \%, 42.5 \%$, and $40 \%$ lower than for FISC. The BER performance of the CESC is quite similar to the performance of the FISC at thresholds of 0.01, 0.001, and 0.0001.

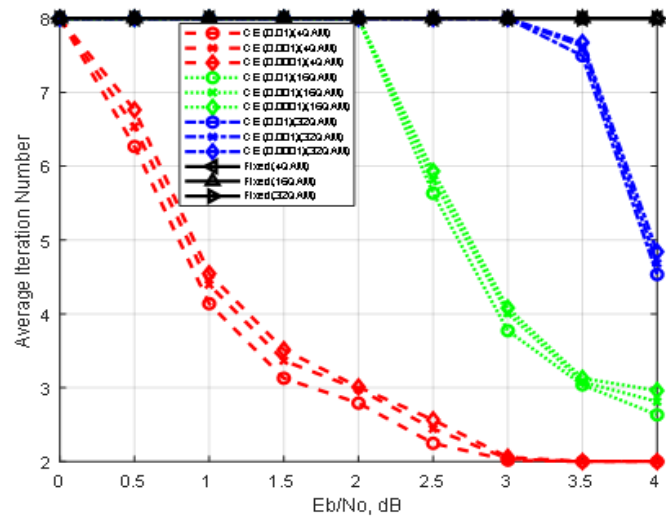

(a)

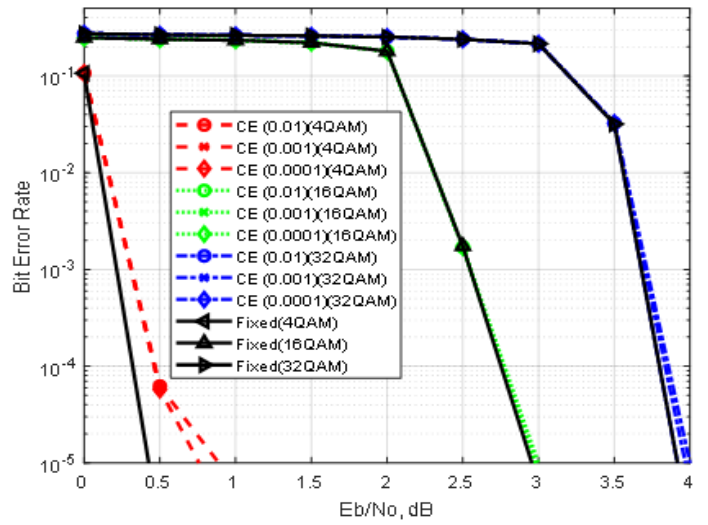

(b)

Figure 4. (a) AIN and (b) BER performances of the CESC with $g=(37,21) \mathrm{R}=1 / 3, \mathrm{~N}=10 \mathrm{~K}$ for different QAM formats

\subsection{Performance of the CESC with different turbo code generators}

The AIN and BER performances of the CESC at different threshold values for different turbo code generators are depicted in Figure 5. The graphs for $\mathrm{g}=(7,5),(17,15)$ and $(37,21)$ show that the AIN for threshold values of $0.01,0.001$ and 0.0001 started to reduce at $2 \mathrm{~dB}$. At $4 \mathrm{~dB} \mathrm{~Eb} / \mathrm{No}$, the threshold values of $0.01,0.001$, and 0.0001 can terminate early at $3 \mathrm{AIN}$, representing savings of around $62.5 \%$ from a FISC. Also, the BER performances of the CESC and FISC are the same for the threshold values of 0.01, 0.001, and 0.0001. 
For $\mathrm{g}=(17,15)$, the threshold values of $0.01,0.001$, and 0.0001 can terminate early at $2.9,3$, and 3 AIN, respectively, representing reductions of around $65 \%, 65 \%$, and $63.75 \%$ compared to FISC. The BER performances of the CESC and FISC were the same at thresholds of 0.01, 0.001, and 0.0001. Meanwhile, for $\mathrm{g}=(37,21)$, at $4 \mathrm{~dB}$, the threshold value of $0.01,0.001$, and 0.0001 can terminate early at $2.8,2.9$, and 3 AIN, respectively, representing savings of around $65 \%, 63.75 \%$, and $62.5 \%$ compared to the FISC. Once again, the CESC's and FISC's BER performances are the same for all three thresholds.

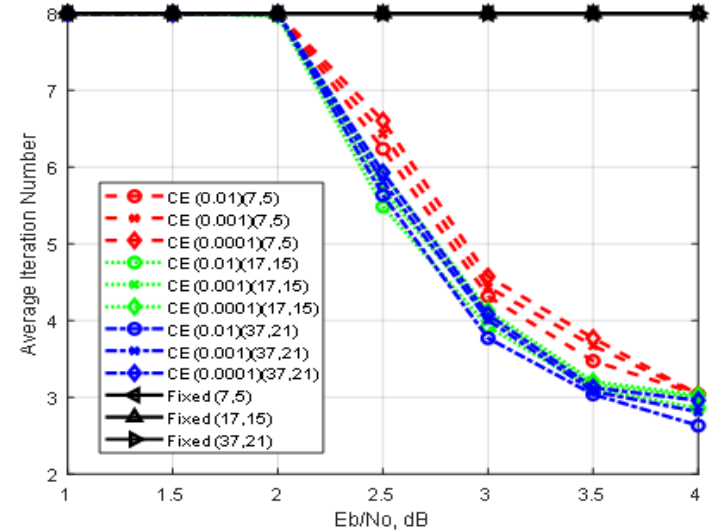

(a)

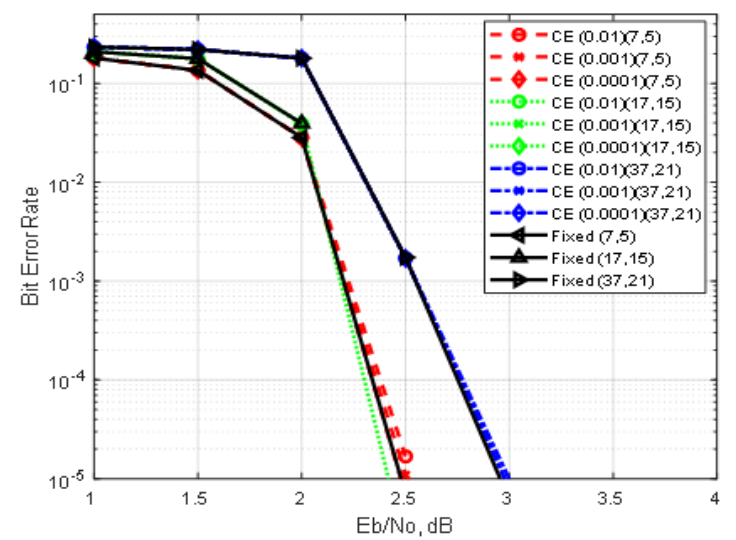

(b)

Figure 5. (a) AIN and (b) BER performances of the CESC with 16-QAM, $R=1 / 3, N=10 \mathrm{~K}$ for different turbo code generators

\subsection{Performance of the CESC with different frame sizes}

The CESC's AIN and BER performances for frame sizes of $1 \mathrm{~K}, 5 \mathrm{~K}$, and $10 \mathrm{~K}$ are illustrated in Figure 6. The $\mathrm{N}=1 \mathrm{~K}$ graph shows that the AIN for threshold values of $0.01,0.001$, and 0.0001 starts to decline at $1.5 \mathrm{~dB}$. At $4 \mathrm{~dB} \mathrm{~Eb} / \mathrm{No}$, the threshold values of $0.01,0.001$, and 0.0001 can terminate early at 2.2, 2.3, and 2.4 AIN, respectively, thus saving around $72.5 \%, 71.25 \%$, and $70 \%$ compared to the FISC. For threshold values of 0.001 and 0.0001, the BER performances of the CESC and FISC are quite close. For threshold $=0.01$, there is a slight indifference in BER it starts at $3 \mathrm{~dB}$ and increases until $4 \mathrm{~dB}$ at $\mathrm{BER}=10^{-4.8}$.

Meanwhile, for $\mathrm{N}=5 \mathrm{~K}$, the $\mathrm{AIN}$ for threshold values of $0.01,0.001$, and 0.0001 starts to reduce at 2 $\mathrm{dB}$. At $4 \mathrm{~dB}$, the threshold values of $0.01,0.001$, and 0.0001 can terminate early at 2.6, 2.8, and 2.9, respectively, thereby saving around $67.5 \%, 65 \%$, and $63.75 \%$ compared to the FISC. Further, the BER performances of the CESC and FISC are the same for thresholds of $0.01,0.001$, and 0.0001 .

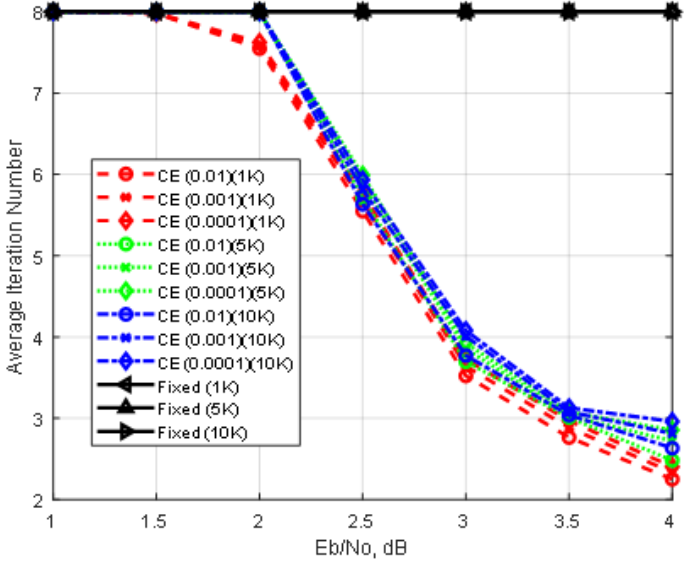

(a)

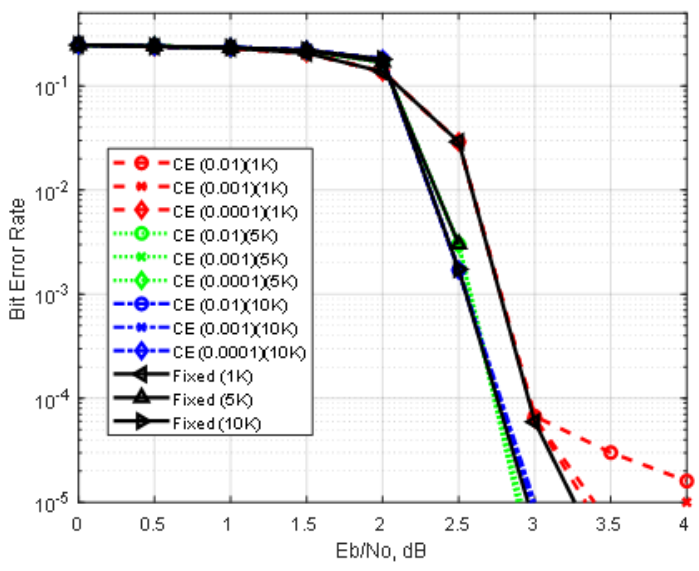

(b0

Figure 6. (a) AIN and (b) BER performances of the CESC with 16-QAM, $g=(37,21), \mathrm{R}=1 / 3$ for different frame sizes 
Finally, for $\mathrm{N}=10 \mathrm{~K}$, the AIN for threshold values of $0.01,0.001$, and 0.0001 start to reduce at $2 \mathrm{~dB}$. At $4 \mathrm{~dB} \mathrm{~Eb} / \mathrm{No}$, the threshold values of $0.01,0.001$ and 0.0001 can terminate early at 2.8, 2.9 and 3.0 AIN, respectively. This early termination represents reductions of around $65 \%, 63.75 \%$, and $62.5 \%$ when compared to the FISC. The BER performances of the CESC and FISC are the same for all three threshold values.

\section{CONCLUSION}

In most situations, the use of the CESC is suitable only when Eb/No or SNR is high. In such cases, the CESC yields significant reductions in AIN while maintaining a desirable BER performance. The CESC can terminate early when Eb/No is high and can save more than $40 \%$ AIN compared with FISC. However, at a low $\mathrm{Eb} / \mathrm{No}$, the CESC fails to terminate early and increases AIN. The CESC's BER performance remains unchanged at BER $>10^{-4}$. However, for the cases of $\mathrm{R}=1 / 2,16-\mathrm{QAM}$ and $\mathrm{R}=1 / 3,4-\mathrm{QAM}$, minor degradations (i.e. of less than $0.2 \mathrm{~dB}$ ) occur in the high $\mathrm{Eb} / \mathrm{No}$ region and increase to $0.5 \mathrm{~dB}$ at $\mathrm{BER} \leq 10^{-4}$. The same situation was apparent for $\mathrm{R}=1 / 3, \mathrm{~N}=1 \mathrm{~K}$, and threshold $=0.01$, as the CESC's BER performance started to decrease after BER $=10^{-4}$. For future works, researchers can use SNR estimations to estimate the low Eb/No region for early CESC termination. In addition, suitable thresholds and enhanced stopping criteria should be calculated and implemented to improve the performance of CESC with QAM for iterative turbo decoding.

\section{ACKNOWLEDGEMENTS}

The authors gratefully acknowledge the support of the Universiti Teknologi MARA (UiTM) for this study, which was funded by grant 600-RMC/MYRA 5/3/LESTARI (039/2020).

\section{REFERENCES}

[1] M. AlMahamdy and J. Dill, "Early Termination of Turbo Decoding by Identification of Undecodable Blocks," in 2017 IEEE Wireless Communications and Networking Conference (WCNC), 2017, pp. 1-6, doi: 10.1109/WCNC.2017.7925644.

[2] H. H. Attar, Ahmad A.A. Solyman, Mohammad R. Khosravi, Lianyong Qi, Mohammad Alhihi and Pooya Tavallali, "Bit and Packet Error Rate Evaluations for Half-Cycle Stage Cooperation on 6G Wireless Networks," Physical Communication, vol. 44, p. 101249, 2021, doi: 10.1016/j.phycom.2020.101249.

[3] M. Zhu, et al., "Combating Error Propagation in Window Decoding of Braided Convolutional Codes," in 2018 IEEE International Symposium on Information Theory (ISIT), 2018, pp. 1380-1384, doi: 10.1109/ISIT.2018.8437819.

[4] Y. Beeharry, T. P. Fowdur and K. M. S. Soyjaudah, "On the Logical Computational Complexity Analysis of Turbo Decoding Algorithms for the LTE Standards," Wireless Personal Communications, pp. 1-29, 2021, doi: 10.1007/s11277-021-08106-x.

[5] M. U. Farooq and M. Lentmaier, "Threshold Computation for Spatially Coupled Turbo-Like Codes on the AWGN Channel," Entropy, vol. 23, no. 2, p. 240, 2021, doi: 10.3390/e23020240.

[6] A. Ouardi, "Effect of Interleaving on The Performances of The MAP Turbo Decoder Controlled by The IHDA and CE Stopping Criteria," Journal of Physics: Conference Series, vol. 1812, no. 1, p. 012006, 2021, doi: 10.1088/1742-6596/1812/1/012006.

[7] R. Mohamad, Harlisya Harun, Makhfudzah Mokhtar, Wan Azizun Wan Adnan and Kaharudin Dimyati, "Threshold-Based Bit Error Rate for Stopping Iterative Turbo Decoding in a Varying SNR Environment," Frequenz, vol. 71, no. 1-2, pp. 83-94, 2017, doi: 10.1515/freq-2016-0018.

[8] A. Hadji, et al., "Shaping duo binary turbo-coded BICM scheme for JPWL image transmission using a link adaptation strategy over wireless channels," Eurasip Journal on Wireless Communications and Networking, vol. 64, no. 1, pp. 1-26, 2021, doi: 10.1186/s13638-021-01949-9.

[9] H. Tawfeek, Gamal M. Abdel-Hamid and Ashraf Mahran, "A Reliability-based Stopping Criterion for Turbo Product Codes," in 2018 14th International Computer Engineering Conference (ICENCO), 2018, pp. 141-145, doi: 10.1109/icenco.2018.8636122.

[10] S. Yoon, Byungkyu Ahn and Jun Heo, "An Advanced Low-Complexity Decoding Algorithm for Turbo Product Codes Based on the Syndrome," Eurasip Journal on Wireless Communications and Networking, vol. 2020, no. 1, pp. 1-31, 2020, doi: 10.1186/s13638-020-01740-2.

[11] R. Mahdi and A. A. Hamad, "Implementation of Efficient Stopping Criteria for Turbo Decoding," Journal of Physics: Conference Series, vol. 1804, no. 1, pp. 1-13, 2021, doi: 10.1088/1742-6596/1804/1/012015.

[12] C.-H. Lin, Shu-Wei Guo, Li-An Ou, "Analysis and Power Evaluation of Window-Stopped Parallel Turbo Decoding for LTE Rate Matching," Iet Communications, vol. 12, no. 9, pp. 1148-1154, 2018, doi: 10.1049/iet-com.2017.1078.

[13] M. Ferrari, S. Bellini and A. Tomasoni, "Safe Early Stopping for Layered LDPC Decoding," IEEE Communications Letters, vol. 19, no. 3, pp. 315-318, 2015, doi: 10.1109/LCOMM.2014.2386853.

[14] T. Fowdur, Y. Beeharry and K. M. S. Soyjaudah, "A Novel Scaling and Early Stopping Mechanism for LTE Turbo Code Based on Regression Analysis," Annals of Telecommunications, vol. 71, no. 7, pp. 369-388, 2016, doi: 10.1007/s12243-016-0514-y. 
[15] R. Xue, Yanbo Sun and Qiang Wei, "Dynamic Iteration Stopping Algorithm for Non-Binary LDPC-Coded HighOrder Prcpm in The Rayleigh Fading Channel," Eurasip Journal on Wireless Communications and Networking, vol. 2016, no. 1, pp. 1-11, 2016, doi: 10.1186/s13638-016-0562-z.

[16] Y. Beeharry, Tulsi Pawan Fowdur and Krishnaraj M. S Soyjaudah, "Performance of Bit Level Decoding Algorithms for Binary LTE Turbo Codes with Early Stopping," Istanbul University-Journal of Electrical \& Electronics Engineering, vol. 17, no. 2, pp. 3399-3415, 2017.

[17] M. Zhu, David G. M. Mitchell; Michael Lentmaier; Daniel J. Costello; Baoming Bai, "Braided Convolutional Codes with Sliding Window Decoding," IEEE Transactions on Communications, vol. 65, no. 9, pp. 3645-3658, 2017, doi: 10.1109/TCOMM.2017.2707073.

[18] M. AlMahamdy and J. Dill, "Half-Iteration Early Termination of Turbo Decoding," in 2017 IEEE 7th Annual Computing and Communication Workshop and Conference (CCWC), 2017, pp. 1-5, doi: 10.1109/CCWC.2017.7868365.

[19] L. O. Mataveli and C. De Almeida, "Complexity Reduction of Convolutional and Turbo Decoding Based on Reliability Thresholds," Wireless Personal Communications, vol. 82, no. 3, pp. 1279-1290, 2015, doi: 10.1007/s11277-015-2282-9.

[20] C. L. Li, Gangfeng Liu, Jie Zhao, "Adaptive Iteration Stopping Criterion for AMSS Equations," in International Conference on Computer Science and Electronic Technology (CSET 2016), 2016, pp. 7-10, doi: 10.2991/cset-16.2016.2.

[21] O. Kocadagli and R. Langari, "Classification of EEG Signals for Epileptic Seizures Using Hybrid Artificial Neural Networks Based Wavelet Transforms and Fuzzy Relations,” Expert Systems with Applications, vol. 88, pp. 419434, 2017, doi: 10.1016/j.eswa.2017.07.020.

[22] N. Gkalelis and V. Mezaris, "Subclass Deep Neural Networks: Re-enabling Neglected Classes in Deep Network Training for Multimedia Classification," in Multimedia Modeling, vol. 11961, Y. M. Ro et al. Eds., (Lecture Notes in Computer Science. Cham: Springer International Publishing Ag, 2020, pp. 227-238, doi: 10.1007/978-3-030-37731-1_19.

[23] M. Bee, Giuseppe Espa,Diego Giuliani and Flavio Santi, "A Cross-Entropy Approach to the Estimation of Generalized Linear Multilevel Models," Journal of Computational and Graphical Statistics, vol. 26, no. 3, pp. 695708, 2017, doi: 10.1080/10618600.2016.1278003.

[24] F. Alberge, "On Some Properties of the Mutual Information Between Extrinsics With Application to Iterative Decoding," IEEE Transactions on Communications, vol. 63, no. 5, pp. 1541-1553, 2015, doi: 10.1109/tcomm.2015.2422293.

[25] M. Krasicki, "Labeling-based Recipient Identification for 16-QAM BICM-ID," Eurasip Journal on Wireless Communications and Networking, vol. 2019, no. 1, pp. 1-9, 2019, doi: 10.1186/s13638-019-1500-7.

[26] M. Y. M. Nasir, Roslina Mohamad, Murizah Kassim, Nooritawati Mat Tahir and Ezmin Abdullah, "Performance Analysis of Cross-Entropy Stopping Criterion for Quadrature Amplitude Modulation," in 2019 IEEE 9th International Conference on System Engineering and Technology (ICSET), 2019, pp. 273-276, doi: 10.1109/ICSEngT.2019.8906345.

\section{BIOGRAPHIES OF AUTHORS}
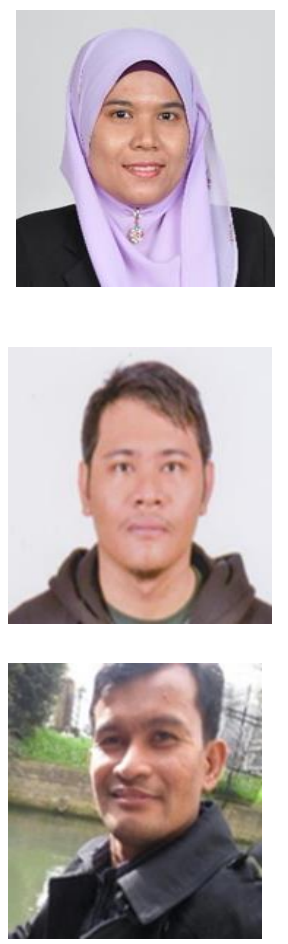

Roslina Mohamad obtained a B. Eng. degree in Electrical Engineering and M. Eng. Science degree from Universiti Malaya, Kuala Lumpur, in 2003 and 2008. She later received a $\mathrm{PhD}$ in Aerospace Engineering from Universiti Putra Malaysia in 2016. Since 2006, she has worked at the School of Electrical Engineering, Universiti Teknologi MARA, as a senior lecturer. Her research interests include computing algorithms and digital signal processing for deep space communication, channel coding, information-theoretic security, computation theory, and wireless communication.

Mohamad Yusuf Mat Nasir obtained a B. Eng. degree in Electronics Engineering from Universiti Teknologi MARA, Shah Alam, in 2017. He is currently pursuing his master's degree at Universiti Teknologi MARA. His research interests include channel coding and wireless communication.

Nuzli Mohamad Anas (SM'14) graduated from Universiti Teknologi Malaysia with a B. Eng. in Electrical Engineering in 2000 and an M. Eng. in Electrical Engineering in 2013. Since 2008, he has worked as a senior researcher at the wireless communication lab at MIMOS Berhad. His research interests include wireless mobile communications and signal processing, especially as it relates to cognitive radio and physical-MAC layer communication design. 